\title{
Anterior cutaneous nerve entrapment syndrome: management challenges
}

This article was published in the following Dove Press journal:

Journal of Pain Research

13 January 2017

Number of times this article has been viewed

\author{
Eleni Chrona ${ }^{1,2}$ \\ Georgia Kostopanagiotou' \\ Dimitrios Damigos ${ }^{3}$ \\ Chrysanthi Batistaki' \\ 'Second Department of \\ Anesthesiology, School of Medicine, \\ National and Kapodistrian \\ University of Athens, "Attikon" \\ Hospital, Athens, 'Department of \\ Anesthesiology, General Hospital \\ of "Ag. Panteleimon," Piraeus, \\ ${ }^{3}$ Department of Medical Psychology, \\ Medical School of loannina, University \\ of Ioannina, loannina, Greece
}

Correspondence: Chrysanthi Batistaki Second Department of Anesthesiology, School of Medicine, National and Kapodistrian University of Athens, Attikon Hospital, I Rimini str,

Athens, 124 62, Greece

Tel +30 2l 0583237 I

Email chrysabatistaki@yahoo.gr

\begin{abstract}
Anterior cutaneous nerve entrapment syndrome (ACNES) is a commonly underdiagnosed and undertreated chronic state of pain. This syndrome is characterized by the entrapment of the cutaneous branches of the lower thoracoabdominal intercostal nerves at the lateral border of the rectus abdominis muscle, which causes severe, often refractory, chronic pain. This narrative review aims to identify the possible therapeutic strategies for the management of the syndrome. Seventeen studies about ACNES therapy were reviewed; of them, 15 were case-control studies, case series, or case reports, and two were randomized controlled trials. The presently available management strategies for ACNES include trigger point injections (diagnostic and therapeutic), ultrasound-guided blocks, chemical neurolysis, and surgical neurectomy, in combination with systemic medication, as well as some emerging techniques, such as radiofrequency ablation and neuromodulation. An increased awareness of the syndrome and the use of specific diagnostic criteria for its recognition are required to facilitate an early and successful management. This review compiles the proposed management strategies for ACNES.
\end{abstract}

Keywords: anterior cutaneous nerve entrapment syndrome, intercostal, neuralgia, management

\section{Introduction}

Chronic abdominal wall pain might have variable etiologies, and thus it requires thorough clinical and laboratory investigations. ${ }^{1,2}$ Abdominal cutaneous nerve entrapment syndrome (ACNES) is one of the causes of chronic abdominal pain, which is mostly underdiagnosed and not appropriately treated. ${ }^{2}$ In this syndrome, the cutaneous branches of the lower thoracoabdominal intercostal nerves are entrapped at the lateral border of the rectus abdominis muscle, resulting in a severe, often refractory, chronic pain. ${ }^{1}$ The prevalence of the syndrome ranges between $15 \%$ and $30 \%$ depending on the definition and the diagnostic criteria used. ${ }^{2-6}$ In adolescents, it is reported to be diagnosed in one out of eight cases of chronic abdominal pain. ${ }^{3,7}$ In the emergency department, the prevalence of ACNES in the patients presenting with acute abdominal pain has been reported to be $2 \% .^{4}$

Anatomically, the lower intercostal nerves (T8-T12) run between the internal oblique and transversus abdominis muscles, up to the point where they reach the rectus abdominis; here, they make a turn in their course to enter the rectus channels (commonly five at each abdominal side). 5,6 Some authors have suggested that the entrapment is usually caused by this sudden turn in their course into the rectus abdominis channel, leading to ACNES. ${ }^{1,7}$ The muscle contraction at this location may cause additional 
nerve compression, and therefore mechanical or ischemic irritation, which results in severe pain. ${ }^{1,2,4}$

The pain is usually localized with a dull and sharp component or may radiate to the ipsilateral half abdomen, following the course of the nerves. ${ }^{1}$ The pain, most of the times, can easily be identified by palpation at the painful site, while a positive Carnett's sign may facilitate the diagnosis. Carnett's sign, named after the scientist who described it in $1926,{ }^{8}$ is when an examiner localizes the exact spot of maximal tenderness on the abdominal wall and asks the patient to raise his head and/or torso with the arms crossed over the chest. ${ }^{1}$ An increased or constant level of pain during this movement, with the examiner's finger in place, indicates that the cause of pain is in the abdominal wall. On the contrary, a negative Carnett's sign indicates a possible visceral cause of the pain., ${ }^{1,4,5,9-21}$

Studies suggest $\mathrm{t}^{4,6,7,10,11,14,19-20}$ some specific diagnostic steps to diagnose ACNES, and these include:

- Unilateral locoregional pain at the abdominal wall lasting for at least 1 month

- The presence of a unilateral tender spot at the abdominal wall (a trigger point of $<2 \mathrm{~cm}^{2}$ fingertip area of maximal tenderness, localized at the lateral border of the rectus abdominis)

- A positive Carnett's test

- A positive skin pinch test and/or altered skin perception to light touch and/or cold at the area of the most intense pain

- Normal laboratory findings with no indication of inflammation or infection, and in the absence of any surgical cause of pain

- Negative imaging of the abdominal wall

- Temporary positive relief in pain response of at least by $50 \%$ after injecting a local anesthetic (usually lidocaine) at the diagnostic trigger point.

Differential diagnosis is very broad and should include all other causes of chronic abdominal pain. ${ }^{1,2,10-12}$ The entrapment or irritation of abdominal cutaneous nerves by scars, postoperative adhesions, or hernias, as well as all other types of neuropathies should be excluded. In most cases, a thorough clinical and laboratory evaluation is required in order to exclude visceral abdominal pathology. ${ }^{1,2,10-12}$

Since ACNES is a relatively underdiagnosed syndrome, the treatment options described in the literature are also limited and not well-defined. The aim of this literature review was to identify and critically evaluate the possible treatment options for ACNES so as to help the clinicians manage this condition effectively.

\section{Literature search strategy}

The authors performed a detailed literature survey in order to identify the studies about the treatment of ACNES in various databases (MEDLINE - PubMed, Scopus, ISI Web of Science, and Google Scholar), using the following keywords (alone or in various combinations): anterior cutaneous nerve entrapment syndrome, ACNES, treatment, management, intercostal neuralgia, chronic abdominal wall pain. Only studies published in English, those that investigated therapeutic options for ACNES, and those that included management as the main outcome measure (in terms of pain intensity and quality, functional status, and/or quality of life) were included. As the literature on this subject is very limited, we considered papers published from 1970 to 2016 .

\section{Outcome of the literature review}

In total, 28 articles that included detailed data on the diagnosis and management of the syndrome were retrieved; however, only 17 studies included detailed information about ACNES therapy. Fifteen of these studies were case-control studies, case series, or case reports, and only two were randomized controlled trials (Table 1).

\section{Systemic drug administration}

Systemic drug administration is usually the first line of treatment for various chronic pain syndromes, ${ }^{12-13}$ including the cases of a nerve injury that causes neuropathic pain. However, in patients with ACNES, the systemic drug therapy is not so effective, mostly as the etiology of nerve irritation is mechanical. All the studies described an initial pain management plan that included systemic drug administration, but without defining details about the exact drugs and the doses used. The most commonly administered drugs were nonsteroidal anti-inflammatory drugs (NSAIDs) such as paracetamol, weak opioids, and drugs generally used for neuropathic pain management, such as antiepileptics (gabapentin and pregabalin) and antidepressants (mostly amitriptyline). ${ }^{12}$ Roderick and Norman ${ }^{13}$ used amitriptyline and lidocaine patches combined with paracetamol and tramadol, which gave good results, while Imajo et al ${ }^{14}$ reported the administration of pregabalin, which was only partially effective. Sahoo and Nair ${ }^{15}$ detailed the administration of NSAIDs, muscle relaxants, tramadol, and gabapentin in two patients, but this did not result in any significant relief. Nizamuddin et al, ${ }^{16}$ who reported the surgical management of three children with ACNES, also mentioned the administration of systemic drugs, such as paracetamol, NSAIDs, 


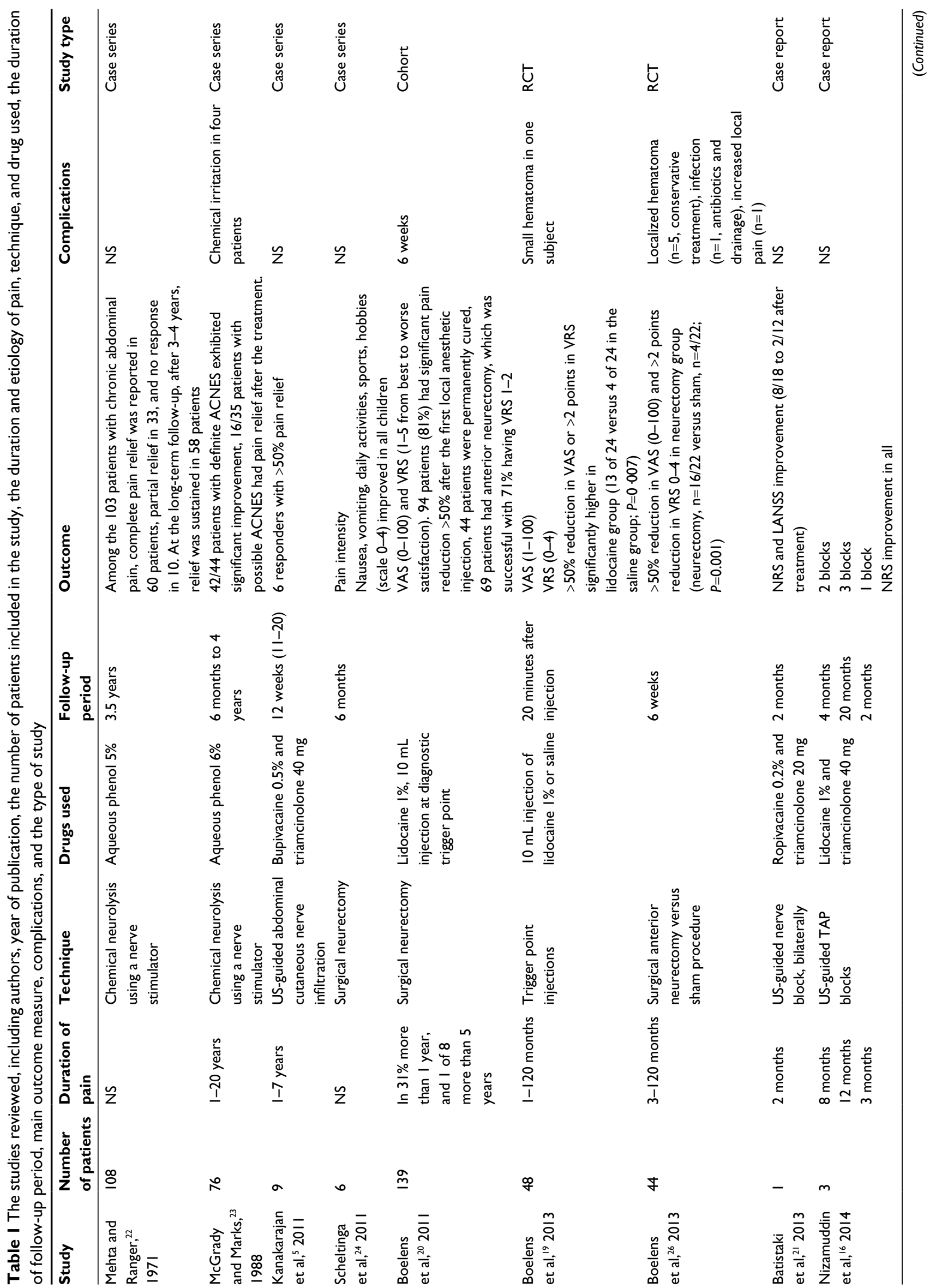




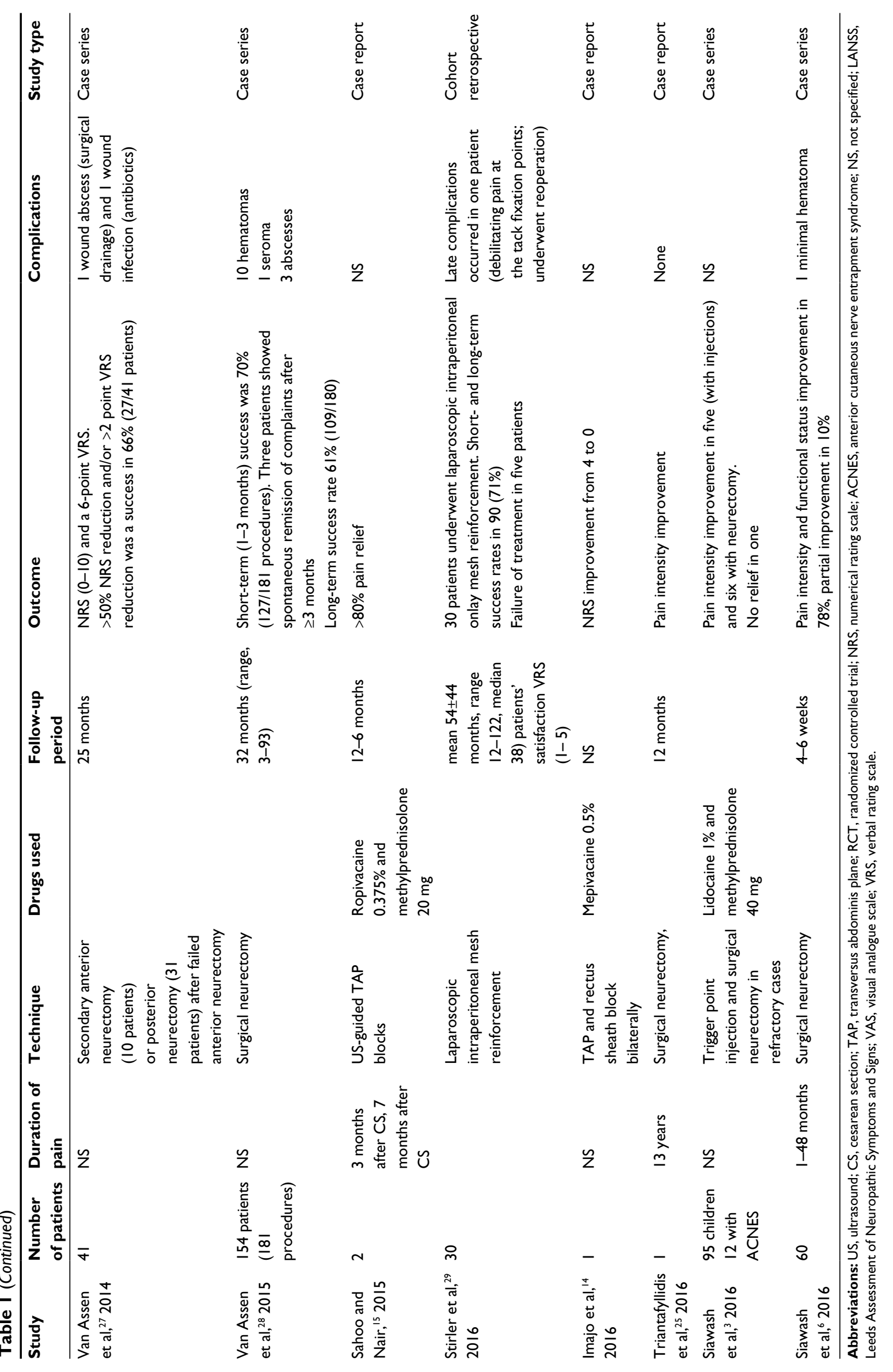


oral opioids, antidepressants, and benzodiazepines (in one patient), before the interventional therapy. However, the systemic therapy was not effective.

Therefore, it is difficult to infer from the current literature if systemic drug administration alone can help patients with ACNES. However, the management of other conditions of chronic pain suggests that systemic drug administration may be used as a first step or in combination with interventional techniques, in order to facilitate patients' recovery and improve their overall quality of life. ${ }^{13,17,18}$ Systemic pharmacotherapy must follow the World Health Organization's analgesic ladder in addition to the guidelines for neuropathic pain management, and this may be used in the course of a multimodal and individualized management plan. ${ }^{17,18}$

\section{Interventional techniques}

Interventional techniques were the main therapeutic option employed in most of the studies on pain management in ACNES. ${ }^{1,2,13,14,17-20}$ These techniques include the trigger point injections (blind or ultrasound-guided), neurolytic techniques (chemical neurolysis or radiofrequency ablation), surgical techniques (anterior neurectomy and mesh reinforcement), and newer techniques such as botulinum toxin injection and neuromodulation. ${ }^{1,2,10-12}$

\section{Trigger point injections}

A specific trigger point on the abdominal wall represents the area of nerve entrapment in most cases, and an injection of local anesthetic at this point could be used for confirmation of ACNES and for therapeutic reasons. ${ }^{1,2,11,19}$ Applegate et al $^{1}$ described the technique in detail using lidocaine (1\%) as a local anesthetic with or without the addition of a corticosteroid. In most cases, the injection was given blindly. This type of injection is based on the loss of resistance as the needle passes through the aponeurosis of the rectus abdominis, ${ }^{1}$ but this blind technique may lead to an incorrect placement of the anesthetic, and it also has the risk of entering into the abdominal cavity. ${ }^{14,19,22}$ More than $50 \%$ pain relief is required to confirm the diagnosis, and in some patients, this effect may last for a variable amount of time - usually significantly longer than the actual half-life of the local anesthetic administered. ${ }^{1}$ The addition of corticosteroids may lead to a more persistent pain relief, possibly owing to its anti-inflammatory action, and the provoked attenuation of ectopic firing. However, their use is often associated with adverse effects, mostly muscle atrophy and systemic adverse reactions. ${ }^{1,19}$ Until now, no direct comparison of lidocaine alone and in combination with a corticosteroid has been carried out for the assessment of their short- and long-term efficacy in the management of ACNES. ${ }^{2}$

On the other hand, trigger point injections with lidocaine alone have been evaluated in a systematic manner. In order to investigate the efficacy of trigger point injections, Boelens et $\mathrm{al}^{20}$ studied 139 patients with ACNES who were diagnosed with an initial injection of $1 \%$ lidocaine $(10 \mathrm{~mL})$ at the point of maximal tenderness. The authors followed a stepwise approach for the management of patients with the syndrome and described the largest database of patients so far. Their data indicated that lidocaine injection resulted in immediate pain reduction in $83 \%$ of the patients with suspected ACNES, and one out of the five patients with a positive result remained persistently pain free. Later, the same team performed a randomized controlled trial in order to test this effect, by comparing the trigger point injection of $1 \%$ lidocaine to saline in 48 patients with ACNES. ${ }^{19}$ Their results revealed a significant difference in the efficacy of lidocaine versus placebo, proving that the local anesthetic itself is involved in pain reduction, and not the injected volume close to the entrapped nerve. However, not all patients responded equally well to lidocaine. This may be attributed to various reasons, especially to the difference in the microanatomy of the painful area. ${ }^{7}$

Siawash et $\mathrm{al}^{6}$ administered a freehand injection to 95 adolescents with chronic abdominal pain for a diagnostic purpose. ACNES was diagnosed only in 12 subjects, who exhibited $>50 \%$ pain relief after receiving the injection of $1 \%$ lidocaine. Three of these patients did not experience pain even after 2 weeks, while other nine required subsequent injections with the addition of methylprednisolone owing to the reappearance of pain. Seven patients did not respond adequately and were scheduled for an anterior neurectomy, which was beneficial in six of them.

Therefore, it can be concluded that the blind trigger point injection technique, both in adults and children, is always diagnostic and sometimes therapeutic, but might also fail due to various reasons. The main reasons for this failure, as described by Koop et al, ${ }^{2}$ include selecting a wrong site for the injection in the blind technique (especially in obese patients or those with some kind of altered anatomy, such as previous surgery, scars, and pregnancy) or owing to a different cause of pain with an incorrect diagnosis.

\section{Ultrasound-guided blocks}

Blindly performed injections have been associated with undesirable results and the injections have a significant risk of entering into the peritoneal cavity. Therefore, ultrasound-guided 
injections have emerged in the last decade. ${ }^{1}$ The main advantages of the ultrasound-guided blocks are the accuracy of injections and the reduction of risk since the injection is performed in real time. Kanakarajan et $\mathrm{al}^{5}$ published a case series of nine patients suffering from ACNES who were diagnosed by clinical symptoms combined with a positive Carnett's sign. All patients were treated with an ultrasound-guided infiltration of the abdominal cutaneous nerves at the site of maximum tenderness and the lateral border of the rectus abdominis muscle. The injected drugs included bupivacaine $(0.5 \%)$ and triamcinolone $(40 \mathrm{mg})$ in a maximum volume of $3 \mathrm{~mL}$. The patients were evaluated using the Brief Pain Inventory as well as the numerical rating scale (NRS), both before the injection and after every 2 weeks for 5 months. Six out of the nine patients got $>50 \%$ pain relief after one injection, while the other three reported less improvement. Among the six responders, five had significant pain relief after only one injection. Batistaki et $\mathrm{a}^{21}$ described a case of bilateral, postsurgical ACNES after a Pfannenstiel incision in a young woman who was treated with ultrasound-guided injections. The nerves' exit point could not be visualized in this patient using the ultrasound, possibly due to the previous surgery and the presence of scars at the abdominal area. In this case, the block was placed at the site of maximum tenderness, under the rectus sheath, along with the rectus abdominis muscle using a larger volume of anesthetic $(10 \mathrm{~mL}$ of $0.2 \%$ ropivacaine combined with triamcinolone). The first injection was diagnostic, but the pain reoccurred, and four more consecutive injections were required to obtain complete pain relief (two with a corticosteroid).

Similarly, Sahoo and Nair ${ }^{15}$ described the successful management of two patients with ACNES using ultrasoundguided transversus abdominis plane (TAP) blocks, injecting a local anesthetic with a corticosteroid. The technique was successful since the block was performed close to the rectus muscle - the anesthetic could diffuse closely to the most possible site of the nerve entrapment. Nizamuddin et al ${ }^{16}$ employed the same technique in children, aiming to perform the block at the closest site to the entrapped nerve, so as to administer smaller volumes of local anesthetics. There were three patients with ACNES (aged 15, 15, and 16 years old), who were treated with ultrasound-guided TAP blocks, using $1 \%$ lidocaine $2-4 \mathrm{~mL}$ and triamcinolone $40 \mathrm{mg}$. One patient had three consecutive blocks, the other had two, and the last one had only one block. The authors suggested that a TAP block guided by ultrasound to the point of maximal tenderness might be the method of choice for approaching the nerve, since it allows visualization of the joint point between the internal oblique and transversus abdominis muscles, thus facilitates injecting the steroid, diluted in smaller volumes of anesthetic closest to the nerve. This is particularly important for the pediatric population. ${ }^{16}$

Finally, yet importantly, Imajo et a ${ }^{14}$ tried a combination of both rectus sheath block and TAP block in a patient with bilateral ACNES that developed after cholecystitis. The authors used $0.5 \%$ mepivacaine without corticosteroid, which provided significant pain relief; however, there are no data on the long-term effect in this patient.

Therefore, from the current literature, it can be concluded that ultrasound-guided techniques using local anesthetics initially and then corticosteroids in refractory states can help in accurate administration of drugs injected and avoid complications. However, no study compares ultrasound-guided and freehand techniques. The ideal ultrasound-guided block for ACNES (trigger point injections around the site of entrapment, rectus sheath block, or TAP block) as well as the ideal type and volume of the drug used (local anesthetic alone or combined with a corticosteroid) have not yet defined. Therefore, future research needs to be directed in this way, as the ultrasound-guided techniques facilitate accurate injections with minimization of side effects.

\section{Chemical neurolysis}

The use of chemical neurolysis, using absolute alcohol or phenol at the exact point of the nerve entrapment, has also been described in the literature as an option for treating ACNES. ${ }^{1}$ However, there are no reports about the long-term effects of this neurodestructive modality, and in the literature, no study carried out in the last decade is available.

In a study by Mehta and Ranger, ${ }^{22}$ a large series of patients with chronic abdominal pain is described. Some of these patients had the characteristics of ACNES, although the syndrome was not described in detail at the time of the study (1971). All patients had a specific tender point of severe pain over the abdominal wall. The authors managed pain with lidocaine injections, along with a corticosteroid (hydrocortisone), and then by neurolysis of the nerve endings using dilute aqueous phenol (5\%) and a neurostimulator to identify the relevant nerve's exit point. Among the 103 patients with chronic abdominal pain, complete pain relief was achieved in 60 patients, partial relief in 33, and there was no response in 10 patients. At a long-term follow-up of 3-4 years, relief was sustained in 58 patients. In this study, no information was given regarding the side effects of the technique or about reoccurrence of pain after a longer period. ${ }^{22}$ 
Similarly, McGrady and Marks, ${ }^{23}$ in another study, followed a protocol of using a nerve stimulator for identifying the entrapped nerve in 76 patients with ACNES, and they employed chemical neurolysis using aqueous $6 \%$ phenol for treatment. Among the 44 patients with ACNES, 42 exhibited significant improvement in their pain after the treatment. On the other hand, among the 35 patients with possible ACNES, only 16 achieved pain relief after the treatment. The mechanism of action of phenol in both the studies could not be explained, since very dilute mixtures were used. According to the authors, a possible mechanism may be the formation of scar tissue around the entrapped nerve, thus preventing its herniation. The pinprick sensation was maintained after the injection. However, no further studies on this technique could be identified in the literature, thus limiting the clinical applicability of this technique.

\section{Surgical techniques Anterior neurectomy}

Surgical anterior neurectomy has been described as a therapeutic option for ACNES in the patients not responding to conservative treatments. Boelens et $\mathrm{al}^{20}$ published the largest database of patients with ACNES who were diagnostically confirmed with the trigger point injection of $1 \%$ lidocaine and corticosteroid at the site of maximal tenderness. In the case of refractory pain, the patients underwent an open anterior neurectomy. Among these patients, 139 were followed by determining the pain score (Visual Analogue Scale [VAS] 0-100 and Verbal Rating Scale [VRS] 1-5) as an outcome measure for long-term efficacy of the technique. The majority of patients (94 patients; $81 \%$ ) had a significant reduction in the pain (of at least $50 \%$ compared to the initial value) after the first local anesthetic injection, and 44 of them achieved permanent relief. In the end, 69 patients underwent anterior neurectomy, which was successful in $71 \%$ of them (VRS ranging between 1 and 2), whereas $9 \%$ of patients showed moderate cure for at least 1 year.

In pediatric cases, Scheltinga et a ${ }^{24}$ reported a case series of eight children with ACNES. Six of them responded only temporarily to lidocaine and corticosteroid injections, and so were treated by anterior neurectomy. The surgical procedure was carried out at the site of maximal tenderness using the Carnett's test, and then the branches of the trapped anterior cutaneous nerve were identified through a $3-5 \mathrm{~cm}$ transverse incision and were removed. All children had a significant improvement in their pain and quality of life as assessed by a five-point Likert scale ( $0=$ never; $4=$ always) regarding the impairment of their daily activities. Another case report of a 13-year-old patient was described by Triantafyllidis et al. ${ }^{25}$ In this patient, surgical neurectomy resulted in a desirable effect. Recently, Siawash et al $^{6}$ published a prospective study of anterior neurectomy in 60 children suffering from ACNES refractory to other treatments. The children were initially treated with a modified rectus sheath block using lidocaine and methylprednisolone. Of the 104 treated children, $66 \mathrm{had}$ refractory pain and were scheduled for anterior neurectomy. Six were excluded from the study due to a previous ACNES surgery, and thus only 60 children could be followed. The pain relief was complete in $78 \%$ of them at the follow-up performed 4-6 weeks after surgery. Another $10 \%$ had only partial pain relief, and $12 \%$ had no relief at all. No other severe complications were reported except one case of minor hematoma.

Boelens et $\mathrm{al}^{26}$ were the first to conduct a randomized controlled trial for the treatment of ACNES with surgical neurectomy employing a sham (placebo) group. They studied patients (aged $>18$ years) diagnosed with ACNES according to specific criteria. ${ }^{20}$ All patients had a diagnostic injection at the relevant trigger point with $1 \%$ lidocaine (positive when $>50 \%$ reduction of pain), but later the relief subsided. The patients were randomly allocated into two groups, with 22 patients in each group. Surgical neurectomy was performed by removal of the neurovascular bundle and all of its branches within a 5-cm radius from the site of fascia opening, while in the sham group only a transverse incision of 5-7 cm, exposing the anterior sheath of the rectus abdominis, was made. The main successful outcome was determined as $>50 \%$ of pain reduction in VAS score $(0-100)$ or $>2$ points in VRS (0-4). Based on this outcome measure, it can be gleaned that the neurectomy patients had significantly more pain relief ( $16 / 22$ neurectomy versus 4/22 sham procedure, with $P=0.001)$ after 6 weeks of follow-up. The 18 patients in the sham group, who had no positive outcome were then reoperated after deblinding, and 11 of them showed a positive response to the treatment. The authors pointed out the necessity of using specific diagnostic criteria for ACNES and recommended a series of injections before the procedure. ${ }^{26}$ However, as mentioned, the surgery was not successful in all patients, which might be due to the anatomic abnormalities leading to the entrapment of the nerve at more dorsal or lateral sites of the rectus abdominis and not at the suspected area, that was the site of the surgery. ${ }^{1,26}$ In these cases, the diagnostic injection was mostly positive, but surgery was not. The authors suggested a surgical exploration of a $5 \times 5$ $\mathrm{cm}$ area around the pain site to identify the possible nerve branches and reduce surgical failure. ${ }^{20}$ 
Van Assen et $\mathrm{al}^{27}$ described the possible options after a failed anterior neurectomy in patients suffering from ACNES. The failed neurectomy was defined as "continued or recurrent pain after the initial procedure". Forty-one patients were reoperated, and a posterior neurectomy and/ or re-exploration of the operated site (secondary anterior neurectomy) was performed. Among the 41 patients (26 with failed neurectomy and 15 with a recurrence of pain), $66 \%$ (27 patients) were significantly improved after the reoperation as followed up to 25 months. Especially the patients who experienced recurrent pain after an initially successful operation had a better outcome compared to the patients in whom the operation was unsuccessful from the beginning. The authors pointed out that approximately one-third of the patients who are operated for ACNES do not respond adequately to this treatment, and the therapeutic options afterward are limited, including mainly systemic pharmacotherapy. Further exploration of the operated area and performing a reoperation may help identify and remove the remaining or persistent nerve endings that cause the pain (secondary anterior neurectomy) or remove the bundle at another level, at the posterior abdominal fascia of the rectus abdominis (posterior neurectomy), leading possibly to a better result. ${ }^{27}$ Data from this study could also be helpful in identifying the success rate of ACNES treatment including various options. The authors presented their data from 181 procedures (154 patients) ${ }^{28}$ and described the treatment efficacy of their stepwise approach: beginning with injections followed by anterior neurectomy in nonresponding cases. Pain scores were recorded using the NRS (0-10) as well as the 6-point verbal category rating scale. In all cases, "success" was defined as a $\geq 50 \%$ reduction in NRS or $\geq 2$ points in category scale. The efficacy of trigger point injections alone was $33 \%$ for long-term pain relief. The success score for neurectomy was $70 \%$ for short-term efficacy (1-3 months), while the overall long-term efficacy was $61 \%$ (after a mean time frame of 32 months). This large database of ACNES patients can be useful in designing a treatment strategy for ACNES. The authors suggested that the initial treatment should be local injections (step 1), followed by anterior neurectomy (step 2), and then reoperation in failed cases (step 3). This approach may achieve up to $90 \%$ success rate, as reported by the study authors..$^{27,28}$

Intraperitoneal onlay mesh reinforcement Since surgery represents a major option of treatment in refractory cases of ACNES, new methods are emerging to provide more long-lasting pain relief. Stirler et al ${ }^{29}$ suggested that intraperitoneal onlay mesh reinforcement may prevent the entrapment of anterior cutaneous nerves of the neurovascular bundle in patients with refractory ACNES. All 40 patients who participated in the study had a prior injection of a local anesthetic combined with a corticosteroid ( $>2$ consecutive injections at the relevant trigger point), but there was no permanent relief in 30 of them. This study presents the results of the technique in those 30 patients who were scheduled for a laparoscopic insertion of polytetrafluoroethylene mesh at the painful area, aiming to overlap the site of pain, covering at least $4 \mathrm{~cm}$ area in all directions. The retrospective analysis of these patients showed excellent short- and long-term success rates of $90 \%$ and $71 \%$, respectively. The patients were evaluated for their satisfaction after the treatment based on a verbal rating on a scale of $1-5$ (with one being the best satisfaction and five the worst; only scores of 1 and 2 were classified as successful treatment). One patient developed pain 1 month after the operation and underwent another operation using the same technique with successful results. The novelty of this technique relies on the fact that neurovascular bundles of the anterior cutaneous nerves emerging from the lower intercostal nerves normally move freely through a fibrous ring in the abdominal wall. In the patients suffering from ACNES, this bundle is compressed and entrapped on this ring. The application of the mesh may decrease the intra-abdominal pressure on the fibrous ring, therefore preventing the entrapment of the nerve and occurrence of pain. This technique needs further assessment through more studies, preferably randomized controlled trials, in order to provide accurate data about its efficacy and longterm outcome.

\section{Other techniques Radiofrequency ablation}

Although there is only one report about the application of radiofrequency ablation for patients with ACNES, ${ }^{30}$ the published studies regarding the other causes of intercostal neuralgia (such as blunt trauma, surgery, and postherpetic neuralgia) reported good results after thermal radiofrequency ablation of the nerve itself or of the relevant dorsal root ganglion (DRG). ${ }^{31-36}$

Tellez Villajos et al, ${ }^{30}$ published a case of ACNES management with radiofrequency ablation of the DRG at the level of T11-T12 with good results. Stolker et al ${ }^{31}$ and van Kleef and Spaans ${ }^{32}$ used pulsed radiofrequency 
in other cases of intercostal neuralgia and observed a significant reduction in pain, while another study reported better results with the pulsed radiofrequency of the DRG. ${ }^{35}$ The classic thermal radiofrequency ablation (performed at $80^{\circ} \mathrm{C}$ for 90 seconds under fluoroscopic guidance and after sensory and motor testing) was also effective in six patients suffering from traumatic intercostal neuralgia from the fourth to the twelfth thoracic nerve level. ${ }^{33}$ In both techniques, postprocedural pain and pneumothorax were the most frequent complications. ${ }^{33}$ There is also another case report presented by Birthi et al, ${ }^{36}$ reporting a patient with chronic abdominal pain who was treated with bilateral pulsed radiofrequency at the level of T10-T11. However, this technique has not been tested in a systematic manner or compared with other treatment options, and its long-term results are unknown.

However, Van Kleef et $\mathrm{al}^{17}$ in their evidence-based guidelines for interventional pain management, rated both the pulsed and the thermal radiofrequency approaches of the DRG in the cases of intercostal neuralgia as $2 \mathrm{C}+$ (effectiveness only demonstrated in observational studies). However, ACNES has a different etiology; the mechanical irritation of the nerve in its peripheral course is the main cause of injury, but it is still an intercostal neuralgia, and the efficacy of radiofrequency ablation in the refractory cases, when other interventional techniques or surgery fails to provide pain relief, is yet to be defined.

\section{Neuromodulation techniques}

The application of neuromodulation techniques, especially peripheral or spinal nerve stimulation has not yet been tried in the therapy of ACNES. However, it might be effective in the management of the disease after the failure of all interventional and surgical therapies, as it has been found to be effective in other forms of neuropathic pain of different origin and etiology. The exact role of nerve stimulation in ACNES is yet to be unraveled, as there is no current evidence to support its use. ${ }^{17,18}$

\section{Botulinum toxin injection}

As an alternative technique, Weum and de Weerd ${ }^{37}$ tried an ultrasound-guided injection of botulinum toxin at the site of maximal tenderness in 15 patients with ACNES. The authors used the Doppler ultrasound and injected $40 \mathrm{IU} / \mathrm{mL}$ of botulinum toxin without local anesthetic in the proximity of the nerve. The number of injections for each patient ranged from 2 to 35 and depended on the duration of the action of botulinum toxin in an individual. The advantages of this technique compared to other ultrasound-guided injections are the localization of the perforator complex at the abdominal wall, the administration of the drug in the proximity to the nerve using the perforator as a guide, and most importantly the drug used. ${ }^{37}$ However, in this study, there were no detailed outcome measures, so it needs to be further evaluated in a more systematic manner.

\section{Discussion}

ACNES is commonly misdiagnosed as the cause of chronic pain. The prevalence of the syndrome is reported to range between $15 \%$ and $30 \%$ in various studies regarding chronic abdominal pain, depending on the definition and the diagnostic criteria used..$^{2-5}$ ACNES should always be included in the differential diagnosis of patients suffering from chronic unilateral abdominal pain, after a thorough clinical and laboratory evaluation. The specific diagnostic steps described in the literature ${ }^{4,9,10,17-20}$ should be followed. No definitive therapy has been prescribed so far, as the literature that supports different types of treatment is mostly based on retrospective case series and a few randomized controlled trials. The main treatment regimen remains therapeutic injections with local anesthetics and corticosteroids, preferably facilitated by ultrasound, and anterior neurectomy in refractory cases. Other options such as neurolysis, radiofrequency ablation, and neuromodulation have not thoroughly been investigated.

The injection of a local anesthetic at the trigger point of the entrapped nerve plays not only a diagnostic role but also a therapeutic one. In most cases, the actual duration of action of the local anesthetic is far longer than the real pharmacological duration of action of the drug, and so patients exhibit pain relief for a long period. ${ }^{10,19,20}$ The addition of corticosteroids has a definite role in the early management of ACNES and should be done preferably under ultrasound guidance to administer the drug as close as possible to the entrapment site..$^{1,2,19,20}$ Although the addition of corticosteroids to the local anesthetic in the case of ACNES has not been investigated in randomized controlled trials, the results from retrospective studies and case series show good results. ${ }^{1,2}$

Regarding the ultrasound-guided techniques, current literature suggests that nowadays blocks should be performed under ultrasound guidance whenever possible. This aims to obtain a more accurate placement of the drugs injected and also to avoid complications. The ideal ultrasound- 
guided block for ACNES has not yet been defined, and many authors have tried different techniques, including trigger point injections, TAP blocks, and rectus sheath blocks. The experience of the practitioner, the anatomic characteristics of each patient, and ability to identify the necessary anatomical structures may all play some role. The altered anatomy of cutaneous nerves traveling across the rectus sheath may have both medial and lateral vascular perforators, as described in detail by Weum and de Weerd. ${ }^{37}$ This is exactly the case where the ultrasound, especially the Doppler, may help visualize the exact exit points of these nerves, a fact that may also help in identifying the area of entrapment of a perforating nerve over the abdomen. Therefore, according to current literature, it seems that ultrasound-guided techniques, whether they are performed at the entrapment site, or more distally, into the rectus sheath or as a TAP block, may be used initially for diagnostic and therapeutic purposes, and can lead in a significant pain relief through an accurate injection of a local anesthetic around the painful nerve.

However, many times, the pain physician needs to manage refractory pain states not responding adequately to initial injections, although the diagnosis may be confirmed. Although chemical neurolysis has been described in the literature, mostly using phenol, and with good results, ${ }^{22,23}$ there are no recent studies on the subject, which limits its clinical applicability at present. More sophisticated techniques, such as radiofrequency ablation, using simple or pulsed radiofrequency, may also have a role in ACNES treatment, but no systematic investigation has been performed thus far. ${ }^{30-34}$ The application of botulinum toxin around the entrapped nerve has also been described in one study and has demonstrated good results, ${ }^{37}$ but no further evidence exists regarding the efficacy of this technique.

In refractory cases, other interventional techniques may be applied, such as open or laparoscopic surgery. The open anterior neurectomy procedure seems to show good results in patients with refractory pain and a definite ACNES diagnosis not responding to therapeutic injections and pharmacotherapy. Most authors agree that the surgical procedure should be restricted to select patients with debilitating, refractory pain and should be performed only at experienced centers. ${ }^{1,19,20,27,28}$ In cases where surgical neurectomy does not relieve pain or leads to only temporary relief with reoccurrence, revision surgery with a posterior approach and re-exploration of the entrapment site have been proposed with good results. ${ }^{27,28}$ According to Van Assen et al, 27,28 the surgeon must be able to identify all possible branches of the entrapped nerves, although the surrounding tissue may appear completely normal. However, these surgical techniques require experienced surgeons with good knowledge of the pathophysiology of the syndrome and the exact anatomy of the area so as to obtain the best outcome for the patient.

Emerging techniques may include radiofrequency ablation in the form of pulsed or thermal radiofrequency, and even neuromodulation techniques, although there is no report in the literature on the subject. The studies based on other forms of neuropathic pain support their use, but only in cases when interventional techniques and/or surgery have proven inadequate. ${ }^{17,18}$ Further research is required, especially in a prospective, randomized manner, to outline the most beneficial therapeutic techniques for ACNES.

A strategy of treatment is proposed in Figure 1. Oor et $a,{ }^{38}$ in their systematic review regarding the treatment of ACNES in adults, described the possible therapeutic techniques, especially trigger point injections, anterior neurectomy, and phenol neurolysis. The authors strongly support trigger point injections as the first-line therapy for the management of ACNES, followed by anterior neurectomy only in severe, refractory cases, and only if all other options have failed.

While compiling this critical review, we faced certain limitations. Most of the studies reviewed are case series or case reports, with limited prospective randomized controlled trials, which limit the strength of evidence for treatment. In addition, many studies originated from the same center, referring to the same database of patients, but describing results obtained from different techniques. A critical evaluation of the studies, but not in a systematic manner, was employed due to the absence of high-quality studies regarding the treatment options of ACNES.

In conclusion, ACNES should always be considered in the differential diagnosis of chronic abdominal pain, and specific diagnostic steps should be followed. The multimodal biopsychosocial approach should never be ignored in all cases, since interventional techniques alone may not be adequate for a successful pain management, especially in persistent refractory cases that have already undergone many treatment plan modifications. It is also wise to consider systemic drug administration following the guidelines for neuropathic pain management, since they may facilitate recovery and further improve patient's quality of life. A multimodal approach is always beneficial in such patients aiming for better long-term recovery. The best approach is yet to be defined, and further research carried out in a more systematic manner employing advanced techniques such as ultrasound-guided procedures is required on the subject. 


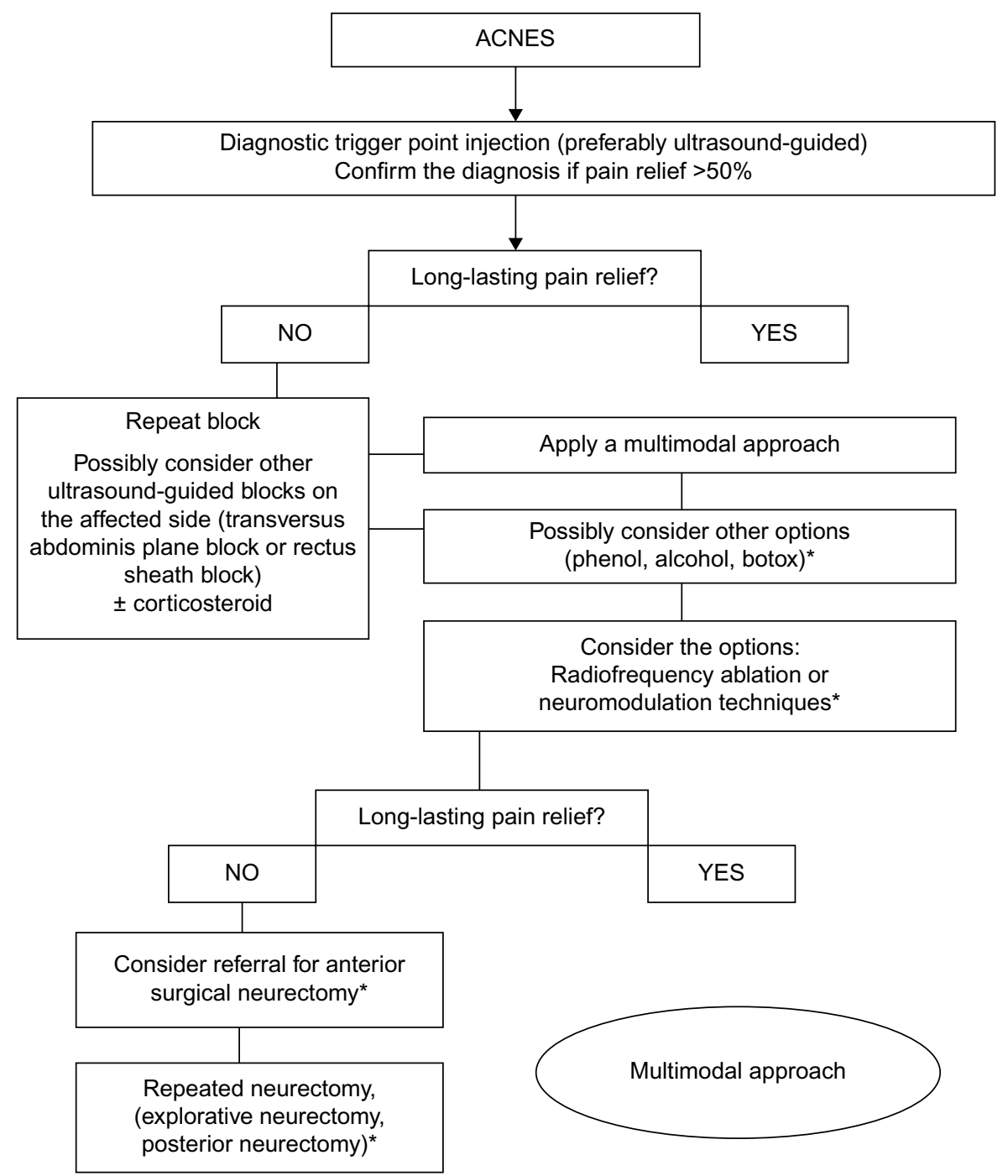

*Only in experienced centers

Figure I Strategy of treatment for ACNES.

Abbreviation: ACNES, anterior cutaneous nerve entrapment syndrome.

\section{Disclosure}

The authors report no conflicts of interest in this work.

\section{References}

1. Applegate WV. Abdominal cutaneous nerve entrapment syndrome (ACNES): a commonly overlooked cause of abdominal pain. Perm J. 2002;6:20-27.

2. Koop H, Koprdova S, Schurmann C. Chronic abdominal wall pain. A poorly recognized clinical problem. Dtsch Arztebl Int. 2016;113(4): 51-57.

3. Siawash M, de Jager-Klevit JW, Ten WT, Roumen RM, Scheltinga MR. Prevalence of anterior cutaneous nerve entrapment syndrome in a pediatric population with chronic abdominal pain. J Pediatr Gastroenterol Nutr. 2016;62(3):399-402.

4. van Assen T, Brouns J, Scheltinga M, Roumen R. Incidence of abdominal pain due to the anterior cutaneous nerve entrapment syndrome in an emergency department. Scand J Trauma Resusc Emerg Med. 2015; 23(19):2-6.
5. Kanakarajan S, High K, Nagaraja R. Chronic abdominal wall pain and ultrasound-guided abdominal cutaneous nerve infiltration. A case series. Pain Med. 2011;12(3):382-386.

6. Siawash M, Maatman R, Tjon A Ten W, van Heurn E, Roumen R, Scheltinga M. Anterior neurectomy in children with a recalcitrant anterior cutaneous nerve entrapment syndrome is safe and successful. J Pediatr Surg. Epub September 1, 2016.

7. Applegate WV, Buchwalter NR. Microanatomy of the structures contributing to abdominal cutaneous nerve entrapment syndrome. $\mathrm{J} \mathrm{Am}$ Board Fam Pract. 1997;10(5):329-332.

8. Carnett JB. Intercostal neuralgia as a cause of abdominal pain and tenderness. Surg Gynecol Obstet.1926;42:625-632.

9. Applegate WV. Abdominal cutaneous nerve entrapment syndrome. Surgery. 1972;71:118-124.

10. van Assen T, Jenneke W, de Jager-Kievit J, Scheltinga M, Roumen R. Chronic abdominal wall pain misdiagnosed as functional abdominal pain. J Am Board Fam Med. 2013;26(6):738-744.

11. Srinivasan R, Greenbaum DS. Chronic abdominal wall pain: a frequently overlooked problem. Practical approach to diagnosis and management. Am J Gastroenterol. 2002;97(4):824-830. 
12. Hooten WM, Timming $\mathrm{R}$, Belgrade $\mathrm{M}$, et al. Institute for Clinical Systems Improvement. Assessment and Management of Chronic Pain. Updated November 2013.

13. Roderick B, Norman B. Anterior cutaneous nerve entrapment syndrome: an unusual cause of abdominal pain during pregnancy. Int J Obstet Anesth. 2016;25:96-97.

14. Imajo Y, Komasawa N, Fujiwara S, Minami T. Transversus abdominal plane and rectus sheath block combination for intractable anterior cutaneous nerve entrapment syndrome after severe cholecystitis. J Clin Anesth. 2016;31:119.

15. Sahoo RK, Nair A. Ultrasound guided transversus abdominis plane block for anterior cutaneous nerve entrapment syndrome. Korean $J$ Pain. 2015;28(4):284-286.

16. Nizamuddin SL, Koury KM, Lau ME, Watt LD, Gulur P. Use of targeted transversus abdominis plane blocks in pediatric patients with anterior cutaneous nerve entrapment syndrome. Pain Physician. 2014; 17(5):E623-E627.

17. Van Kleef M, Stolker R, Lataster A, Geurts J, Benzon H, Mekhail N. Thoracic pain. In: Van Zundert J, editor. Evidence-Based Interventional Pain Management: According to Clinical Diagnosis. Chichester, UK: Wiley-Blackwell; 2012:62-70.

18. Rosati C, Stain S. Abdominal pain. In: Smith H, editor. Current Therapy in Pain. Philadelphia PA: Saunders; 2009:201-204.

19. Boelens OB, Scheltinga MR, Houterman S, Roumen RM. Randomized clinical trial of trigger point infiltration with lidocaine to diagnose anterior cutaneous nerve entrapment syndrome. Br J Surgery. 2013;100(2):217-221.

20. Boelens OB, Scheltinga MR, Houterman S, Roumen RM. Management of anterior cutaneous nerve entrapment syndrome in a cohort of 139 patients. Ann Surg. 2011;254(6):1054-1058.

21. Batistaki C, Saranteas T, Adoni A, Kostopanagiotou G. Ultrasoundguided anterior cutaneous nerve block for the management of bilateral abdominal cutaneous nerve entrapment syndrome (ACNES). Pain Physician. 2013;16(6):E799-E801.

22. Mehta M, Ranger I. Persistent abdominal pain. Treatment by nerve block. Anaesthesia. 1971;26(3):330-333.

23. McGrady EM, Marks RL. Treatment of abdominal nerve entrapment syndrome using a nerve stimulator. Ann R Coll Surg Engl. 1988; 70(3):120-122.

24. Scheltinga MR, Boelens OB, Tjon WE, Ten TA, Roumen RM. Surgery for refractory anterior cutaneous nerve entrapment syndrome (ACNES) in children. J Pediatr Surg. 2011;46(4):699-703.

25. Triantafyllidis A, Mosharaf A, Low H. Anterior cutaneous nerve entrapment syndrome following an appendectomy. Case report. Neurol Sci. 2016;37(5):823-824.
26. Boelens OB, van Assen T, Houterman S, Scheltinga MR, Roumen RM. A double-blind, randomized, controlled trial on surgery for chronic abdominal pain due to anterior cutaneous nerve entrapment syndrome. Ann Surg. 2013;257(5):845-849.

27. Van Assen T, Boelens OB, van Eerten PV, Scheltinga MR, Roumen RM. Surgical options after a failed neurectomy in anterior cutaneous nerve entrapment syndrome. World J Surg. 2014;38(12):3105-3111.

28. Van Assen T, Boelens OB, van Eerten PV, Perquin C, Scheltinga MR, Roumen RM. Long-term success rates after an anterior neurectomy in patients with an abdominal cutaneous nerve entrapment syndrome. Surgery. 2015;157(1):137-143.

29. Stirler MA, Raymakers JT, Rakic S. Intraperitoneal onlay mesh reinforcement of the abdominal wall: a new surgical option for treatment of anterior cutaneous nerve entrapment syndrome - a retrospective cohort analysis of 30 consecutive patients. Surg Endosc. 2016; 30(7):2711-2715.

30. Téllez Villajos L, Hinojal Olmedillo B, Moreira Vicente V, de la Calle Reviriego JL, Senosiain Lalastra C, Foruny Olcina JR. Radiofrecuencia pulsada en el tratamiento del síndrome de atrapamiento del nervio cutáneo abdominal. Gastroenterol Hepatol. 2015;38(1):14-16.

31. Stolker RJ, Vervest ACM, Groen GJ. The treatment of chronic thoracic segmental pain by radiofrequency percutaneous partial rhizotomy. J Neurosurg. 1994;80(6):986-992.

32. van Kleef M, Spaans F. The effects of producing a radiofrequency lesion adjacent to the dorsal root ganglion in patients with thoracic segmental pain by radiofrequency percutaneous partial rhizotomy. Clin J Pain. 1995;11(4):325-332.

33. Engel A. Utility of intercostal nerve conventional thermal radiofrequency ablations in the injured worker after blunt trauma. Pain Physician. 2012;15(5):E711-E718.

34. Akkava T, Ozkan D. Ultrasound-guided pulsed radiofrequency treatment of the intercostal nerve: three cases. J Anesth. 2013;27(6): 968-969.

35. Cohen SP, Sireci A, Wu CL, Larkin TM, Williams KA, Hurley RW. Pulsed radiofrequency of the dorsal root ganglia is superior to pharmacotherapy or pulsed radiofrequency of the intercostals nerves in the treatment of chronic postsurgical thoracic pain. Pain Physician. 2006; 9(3):227-235.

36. Birthi P, Calhoun D, Grider J. Pulsed radiofrequency for chronic abdominal pain. Pain Physician. 2013;16:E443-E450.

37. Weum $\mathrm{S}$, de Weerd L. Perforator-guided drug injection in the treatment of abdominal wall pain. Pain Med. 2016;17(7):1229-1232.

38. Oor JE, Ünlü Ç, Hazebroek EJ. A systematic review of the treatment for Abdominal Cutaneous Nerve Entrapment Syndrome (ACNES). Am J Surg. 2016;212(1):165-174.
Journal of Pain Research

\section{Publish your work in this journal}

The Journal of Pain Research is an international, peer reviewed, open access, online journal that welcomes laboratory and clinical findings in the fields of pain research and the prevention and management of pain. Original research, reviews, symposium reports, hypothesis formation and commentaries are all considered for publication.

\section{Dovepress}

The manuscript management system is completely online and includes a very quick and fair peer-review system, which is all easy to use. Visit http://www.dovepress.com/testimonials.php to read real quotes from published authors. 